\title{
ANALYTICITY OF HOMOLOGY CLASSES
}

\author{
ALBERTO TOGNOLI
}

(Communicated by Haynes R. Miller)

\begin{abstract}
Let $W$ be a real analytic manifold and $\{\alpha\} \in H_{p}\left(W, Z_{2}\right)$. We shall say that $\{\alpha\}$ is analytic if there exists a compact analytic subset $S$ of $W$, such that: $\{\alpha\}=\{$ fundamental class of $S\}$. The purpose of this short paper is to prove

THEOREM 1. Let $W$ be a paracompact real analytic manifold; then any homology class $\{\alpha\} \in H_{p}\left(W, Z_{2}\right)$ is analytic.

We remember that a similar result does not hold in the real algebraic case (see [1]).
\end{abstract}

1. Definitions and well-known facts. Let $V, W$ be two differentiable (i.e. $\left.C^{\infty}\right)$ manifolds; then, on the set $M(V, W)$ of differentiable maps $f: V \rightarrow W$, we shall consider the Whitney topology (see [2, p. 42]). In the following we shall use the known result: if $f \in M(V, W)$, then there exists a neighborhood $U$, in the $C^{0}$ topology, of $f$ such that any $g \in U$ is homotopic to $f$ (see [8]). By real algebraic variety we shall mean: affine real algebraic variety. A regular algebraic variety shall be called: algebraic manifold. An algebraic map is the restriction of a rational regular map.

In the following we shall need

LEMMA 1. Let $V \subset \mathbf{R}^{n}, W \subset \mathbf{R}^{q}$ be two real algebraic manifolds and $V \stackrel{\varphi}{\longrightarrow} W$ a differentiable map. If $V$ is compact and boarding to $\phi$, then, for any $\varepsilon>0$, there exists an algebraic submanifold $V^{\prime} \subset \mathbf{R}^{n+q}$, an analytic isomorphism $V^{\prime} \stackrel{\pi}{\rightarrow} V$ and an algebraic map $\varphi^{\prime}: V^{\prime} \rightarrow W$ such that

(i) $\delta\left(\varphi(x), \varphi^{\prime} \circ \pi^{-1}(x)\right)<\varepsilon, x \in V$,

(ii) $\delta^{\prime}\left((d \varphi)(v),\left(d\left(\varphi^{\prime} \circ \pi^{-1}\right)\right)(v)\right)<\varepsilon$

for any tangent vector $v$, to $V$ in $x$, where $\delta, \delta^{\prime}$ are two metrics on $\mathbf{R}^{q}$ and on the Grassmannian manifold.

ProOF. See [3].

LEMMA 2. Let $V \subset \mathbf{R}^{n}, W \subset \mathbf{R}^{q}$ be two real algebraic manifolds and $\varphi: V \rightarrow$ $W$ an algebraic map. Let us suppose that $V$ is irreducible and there exists a Zariski open set $V^{\prime} \subset V$ with the property: $\varphi$ is injective on $V^{\prime}$. Under these hypotheses, if $T$ is the Zariski closure of $\varphi(V)$ in $W$, we have

(i) $T \supset \varphi(V), \operatorname{dim} T=\operatorname{dim} V ; T-\varphi(V)$ is contained in an algebraic set $S$, $\operatorname{dim} S<\operatorname{dim} V$,

(ii) $\{$ fundamental class of $T\}=\varphi_{*}$ \{fundamental class of $\left.V\right\}$.

Received by the editors November 22, 1986.

1980 Mathematics Subject Classification (1985 Revision). Primary 55M57; Secondary 14 G58. 
ProOF. (i) is proved in [4, Lemma 1.1]. (ii) follows from the definition of the fundamental class (see [5]) and the proof of the first part of the lemma.

Now let $\varphi: V \rightarrow W$ be a differentiable map between differentiable manifolds. Let us denote by $\mathscr{E}(V)_{x}, \mathscr{E}(W)_{\varphi(x)}$ the stalks of the sheaves of the differentiable functions on $V, W$. We recall

DEFINITION. $\varphi$ is called finite, in the point $x$, if $\mathscr{E}(V)_{x}$ is a finite $\varphi^{*}\left(\mathscr{E}(W)_{\varphi(x)}\right)$ module.

We have

LEMMA 3. Let $\operatorname{dim} V \leq \operatorname{dim} W$ and let us suppose that $V$ is compact. Then the set of differentiable maps that are finite in any point is an open dense subset of $M(V, W)$.

ProOF. See [6, p. 96] (see also [2, p. 169]).

2. The proof of the theorem. Now let us suppose that $W$ is a compact real analytic manifold and $\{\alpha\} \in H_{p}\left(W, Z_{2}\right)$. It is known (see [7]) that we may suppose $W$ is a real algebraic manifold. Moreover, there exists a compact differentiable manifold $V$ and a differentiable map $\varphi: V \rightarrow W$ such that $\{\alpha\}=\varphi$ (fundamental class of $V$ ) (see [8]). By Lemma 1 we may suppose there exists an algebraic manifold $\widehat{V}=V^{\prime} \cup V^{\prime \prime}$ and an algebraic map $\hat{\varphi}: \widehat{V} \rightarrow W$ such that:

(i) $V^{\prime}$ and $V^{\prime \prime}$ are diffeomorphic to $V$,

(ii) $\{\alpha\}=\hat{\varphi}_{*}$ (fundamental class of $\left.V^{\prime}\right)=\hat{\varphi}$ (fundamental class of $V^{\prime \prime}$ ),

(iii) $\left.\hat{\varphi}\right|_{V^{\prime}}$ is in general position with respect to $\hat{\varphi}\left(V^{\prime \prime}\right)$.

Moreover, by Lemma 3, we may suppose

(iv) $\hat{\varphi}$ is finite in any $x \in \widehat{V}$.

From Lemma 2, we may finally assume that

(v) if $T$ is the Zariski closure of $\hat{\varphi}(\widehat{V})$ in $W$, then $T-\hat{\varphi}(\widehat{V})$ is contained in an algebraic set $S$ such that $\operatorname{dim} S<\operatorname{dim} V=p$.

Let now $\tilde{\hat{\varphi}}: \widetilde{\widehat{V}} \rightarrow \widetilde{W}$ be a complexification of $\hat{\varphi}$, such $\tilde{\hat{\varphi}}$ exists (see [9]), and we may suppose $\tilde{\hat{\varphi}}$ is finite in any point of $\widetilde{\widehat{V}}$, because the finiteness is an open condition (see [2, p. 168]). We shall suppose that $\widetilde{\widehat{V}}=\widetilde{V}^{\prime} \amalg \widetilde{V}^{\prime \prime}$. The map $\tilde{\hat{\varphi}}$ is finite, hence the image of any analytic germ $\widetilde{\widehat{V}}_{y}$ is the germ of a complex analytic set of $\tilde{W}$, see [10, p. 162].

Let us now remark that the above facts imply that:

(a) $T=$ real part of the closure in the Zariski topology of $\tilde{\hat{\varphi}}(\widetilde{\widehat{V}})$,

(b) $d \tilde{\hat{\varphi}}$ has maximum rank on an open dense set of $\widetilde{\hat{V}}$.

We deduce that, for any $x \in T$, we have three disjoint analytic irreducible germs of $T_{x}$ :

(1) germs image of $\tilde{V}^{\prime}$, of dimension $p$,

(2) germs image of $\tilde{V}^{\prime \prime}$, of dimension $p$,

(3) germ image of $\tilde{\hat{\varphi}}^{-1}(S)$, of dimension lower than $p$.

This proves that $T^{\prime}=\hat{\varphi}\left(V^{\prime}\right) \cup S$ is an analytic subset of $W$ and, clearly,

$$
\{\alpha\}=\left\{\text { fundamental class of } T^{\prime}\right\} .
$$


In fact, for any $y \in T$, the germ $Y_{y}=\left|\bigcup_{i} \tilde{\hat{\varphi}}\left(\widetilde{V}_{y_{i}}^{\prime}\right)\right|_{R}$ is real analytic, where $\bigcup y_{i}=\tilde{\hat{\varphi}}^{-1}(y) \cap \tilde{V}^{\prime},|Z|_{R}=$ real part of $Z$.

So $Y_{y} \cup S_{y}$ is real analytic and clearly $Y_{y} \cup S_{y}=T_{y}^{\prime}$. In fact, in any point, $T^{\prime}$ is the union of a finite set of irreducible germs of $T$. So the theorem is proved under the hypothesis: $W$ is compact. In the general case, let us remark that we may take a representative element $\alpha$ of $\{\alpha\}$ contained in a relatively compact open set $U$ of $W$. We can now see $U$, up to analytic isomorphism, as an open set of a compact analytic manifold $Z$ (take the unique analytic structure on the double of $U$ ). We can now prove the analyticity of $\{\alpha\}$ in $Z$ and this implies, clearly, the analyticity of $\{\alpha\}$ in $W$. The theorem is proved.

\section{BIBLIOGRAPHY}

1. R. Benedetti and M. Dedo, Counterexamples to representing homology classes by real algebraic varieties up to homeomorphisms, Compositio Math. 53 (1984).

2. M. Golubisky and V. Guillemin, Stable mappings and their singularities, Springer-Verlag, Berlin and New York, 1973.

3. A. Tognoli, Algebraic approximation of manifolds and spaces, Sem. Bourbaki 548 (1979-80).

4. R. Benedetti and A. Tognoli, On real algebraic vector bundles, Bull. Sci. Math. (2) 104 (1980).

5. A. Borel and A. Haefliger, La classe d'homologie fondamentale d'une espace analytique, Bull. Soc. Math. France 89 (1961).

6. A. Andreotti and P. Holm, Quasianalytic and parametric spaces, Real and Complex Singularities, Noordhoff, Oslo, 1976.

7. A. Tognoli, Su una congettura di Nash, Ann. Scuola Norm. Sup. Cl. Sci. Pisa (3) 27 (1973).

8. R. Thom, Quelques propriétés globales des variétés différentiables, Comment. Math. Helv. 28 (1954).

9. A. Tognoli, Algebraic geometry and Nash functions, Institutiones 3 (1978),.

10. L. Kaup and B. Kaup, Holomorphic functions of several variables, De Gruyter, 1983.

Istituto Matematico, Università di Trento, Trento, Italy 